Karger Kompass Ophthalmol 2019;5:194-197

\title{
Spektrum Ophthalmologie - wissenswert, kompakt, anregend
}

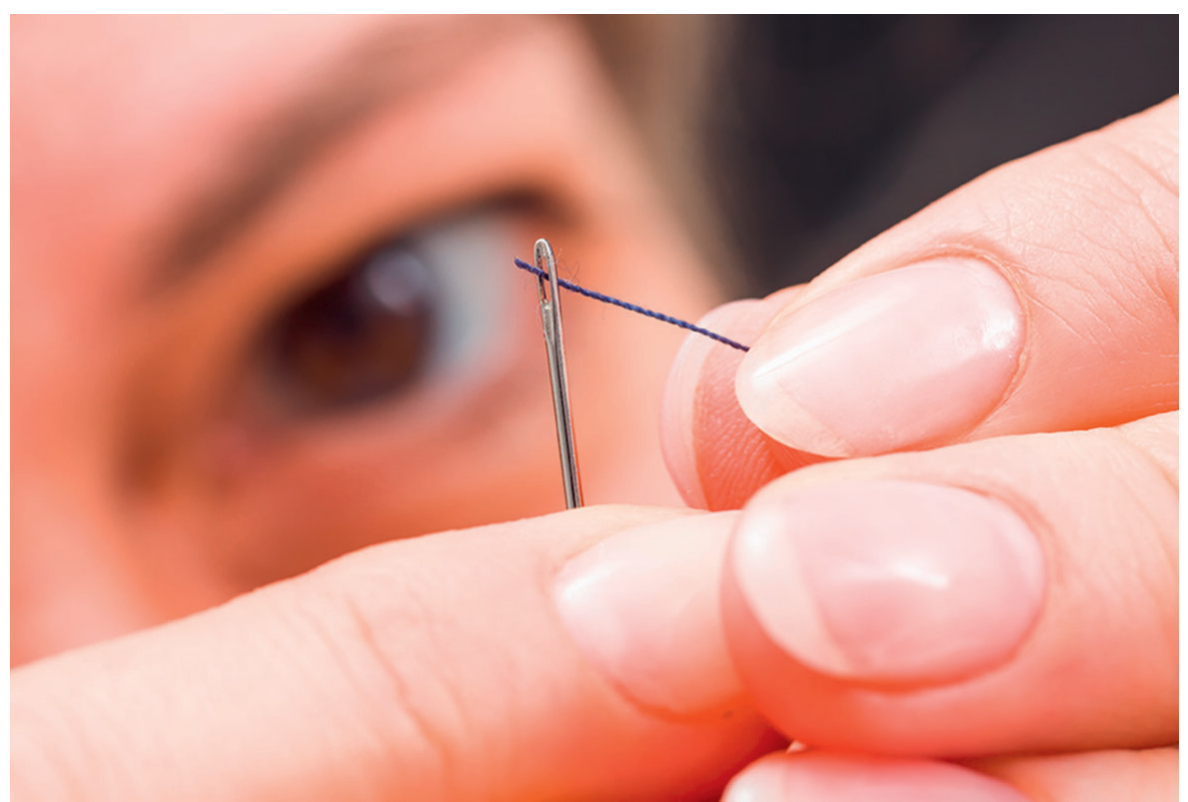

(c) Adobe Stock

Hertie-Institut für klinische Hirnforschung (HIH)

Wahrnehmungssteuerung: Wie das «Obere Hügelchen» im Gehirn hilft, eine Nadel einzufädeln

Wir sehen nur rund 1-2\% unserer Umwelt wirklich scharf-und zwar den Ausschnitt, der in die Sehgrube (lat:: Fovea Centralis) der Netzhaut fällt. Um knifflige Handlungen auszuführen, wie etwa einen Faden durch ein Nadelöhr zu ziehen, müssen unsere Augen daher viele kleine und sehr präzise Bewegungen ausführen. Doch wie kontrolliert unser Gehirn diese Bewegungen? Dieser Frage sind Neurowissenschaftler um Prof. Ziad M. Hafed am Hertie-Institut für klinische Hirnforschung und der Universität Tübingen nachgegangen. Ihren Ergebnissen zufolge spielt dabei das sogenannte Obere Hügelchen (lat::Superior Colliculum) im Hirnstamm die Hauptrolle. «30-40\% der dortigen Nervenzellen verarbeiten 1-2\% des visuellen Bildes», so Hafed.
Die Zahl der Nervenzellen, die an der Verarbeitung von hochaufgelösten Bildbereichen beteiligt sind, vergrößert sich also drastisch. Seine Schlussfolgerung: «Damit eignet sich das Obere Hügelchen perfekt, um auch präzise Augenbewegungen zu lenken.» Das stelle einen Wendepunkt für die Sehforschung dar: Bislang sei man davon ausgegangen, dass das Obere Hügelchen nur dazu notwendig ist, die Augen aus dem Bereich des schärfsten Sehens wegzubewegen.

Die Entschlüsselung der Sehverarbeitung in tieferen Hirnstrukturen hilft Hirnforschern auch, bestimmte Sehstörungen besser zu verstehen. So ist beim sogenannten Blindsehen die primäre visuelle Großhirnrinde etwa durch einen Schlaganfall verletzt. Diese Pati- enten sind erblindet, können jedoch auf manche visuellen Reize unbewusst reagieren und z.B. einen heranfliegenden Ball fangen oder inm ausweichen. «Parallele Sehbahnen, wie sie etwa über das Obere Hügelchen laufen, verleihen hier die Restfähigkeit», erklärt Hafed. «Erkenntnisse über diese Wege können in Zukunft genutzt werden, um sie spezifisch zu stimulieren und dadurch spezielle Sehprobleme zu lindern».

Literatur

1 Chen et al. Current Biology 2019;29:1-11.

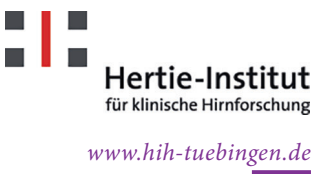

\section{KARGER}

(c) 2019 S. Karger GmbH, Freiburg 


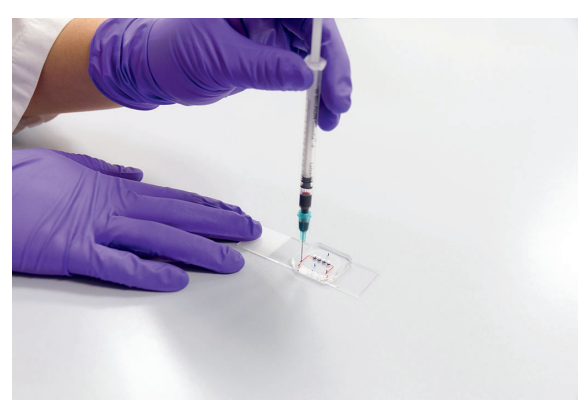

Anordnung des Organ-on-a-Chip-Systems. (c) Fraunhofer-IGB

Schädigungen der Netzhaut sind häufig integraler Bestandteil von Erkrankungen, die zu Blindheit führen. Die Netzhaut ist darüber hinaus anfällig für schädigende Nebenwirkungen von Medikamenten.

Bisherige Modelle zur Untersuchung von Augenkrankheiten und Nebenwirkungen von Medikamenten können die Physiologie der menschlichen Netzhaut nicht oder nur ansatzweise widerspiegeln. Einer For- schungsgruppe des Fraunhofer-Instituts für Grenzflächen- und Bioverfahrenstechnik (IGB) in Stuttgart ist nun die Integration lebender menschlicher Zellen in ein künstliches mikrophysiologisches System gelungen.

«Mit unserer Retina-on-a-Chip können wir erstmals ein dreidimensionales Netzhautmodell vorweisen, das einen Großteil der strukturellen Merkmale und Funktionalität der menschlichen Netzhaut nachbildet», so Dr. Kevin Achberger, Postdoc am Lehrstuhl für Neuroanatomie und Entwicklungsbiologie der Eberhard Karls Universität Tübingen.

«Ein Vorteil dieses winzigen Modells ist, dass es als Teil eines automatisierten Systems verwendet werden könnte, um Hunderte von Medikamenten sehr schnell auf schädigende Wirkungen auf die Netzhaut zu testen. Außerdem ermöglicht es Wissenschaftlern, Stammzellen eines bestimmten Patienten zu entnehmen und sowohl die
Krankheit als auch mögliche individuelle Behandlungen zu untersuchen.»

«Dieser neue Ansatz kombiniert zwei vielversprechende Technologien - Organoide und Organ-on-a-Chip - und hat das Potenzial, die Medikamentenentwicklung zu revolutionieren und eine neue Ära der personalisierten Medizin einzuleiten», sagt Peter Loskill, Juniorprofessor für Experimentelle Regenerative Medizin an der Eberhard Karls Universität Tübingen und Leiter der AttractGruppe Organ-on-a-Chip am Fraunhofer IGB. Sein Labor hat bereits ähnliche Organon-a-Chip-Systeme für Herzmuskel, Fettgewebe und Bauchspeicheldrüse entwickelt.

\section{Literatur}

Achberger K, et al.: eLife 2019;8:e46188

Fraunhofer IGB

www.igb.fraunhofer.de

\section{Deutsche Gesellschaft für Neurologie \\ Seltene Ursache von Sehverlust und Querschnittslähmung besser behandelbar}

Erkrankungen des Neuromyelitis-opticaSpektrums (NMOSD) sind seltene Autoimmunerkrankungen des zentralen Nervensystems [1, 2]. Dabei greifen Zellen des Immunsystems fälschlicherweise die eigenen Nervenzellen der Sehnerven und des Rückenmarkes an. Es kommt zu Entzündungen der Sehnerven mit Sehstörungen bis hin zur Erblindung. Die Erkrankung verläuft in der Regel schubförmig. Bei rechtzeitiger Behandlung eines Schubes (z.B. mit Kortison) kann sich die Symptomatik vollständig zurückbilden; bei unvollständiger Erholung kommt es jedoch zu bleibenden Behinderungen, die bei jedem Schub fortschreiten. Die beste Behandlung ist neben der schnellstmöglichen Schubtherapie die Prophylaxe neuer Schübe. Dies erfolgt medikamentös durch eine dauerhafte Immunsuppression. So wird zurzeit erfolgreich Rituximab zur NMO-Schubprophylaxe eingesetzt.
Bei der Suche nach wirksamen NMO-Therapien ist auch Eculizumab von großem Interesse, da seit fast 20 Jahren die Rolle der Komplementaktivierung bei der NMO bekannt ist. Es zeigte ein relativ gutes Sicherheitsprofil, jedoch klagten mehr Patienten über Kopfschmerzen und obere Atemwegsinfekte, ein Patient verstarb an einer Infektion der Lunge [3].

PD Dr. Harald Prüß von der Klinik für Neurologie der Charité und dem Deutschen Zentrum für Neurodegenerative Erkrankungen e. V. (DZNE) in Berlin erläutert aber auch die Limitationen der Studie: «Angesichts der Schwere der NMO-Erkrankung war eine Placebogruppe ohne jegliche Behandlung ethisch nicht vertretbar, sodass die Mehrzahl der Patienten beider Gruppen noch weitere Immuntherapien erhielt - diese könnten Effekte auf das Studienergebnis gehabt haben, letztlich auch auf die Nebenwirkungsrate.»
Eculizumab muss alle zwei Wochen als Infusion verabreicht werden, was die Anwendung in der klinischen Routine erschwert. Hinzu kommen die sehr hohen Kosten des Medikamentes. «Dennoch kann Eculizumab zu einem sehr wichtigen Reservemedikament für die NMO werden und die Patienten vor bleibender Behinderung retten, wenn die Standardtherapie, nicht ausreicht.»

\section{Literatur}

1 https://nemos-net.de/fuer-patienten-was-ist-dienmo.html

2 https://www.dgn.org/neuronews/3159-schubtherapie-der-neuromyelitis-optica-plasmaaustauschverfahren-besser-als-steroide

3 Pittock SJ et al. N Engl J Med 2019; 381 (7): 614-625

\section{DGNv}




\section{Augenscan macht Krankheiten frühzeitig sichtbar}

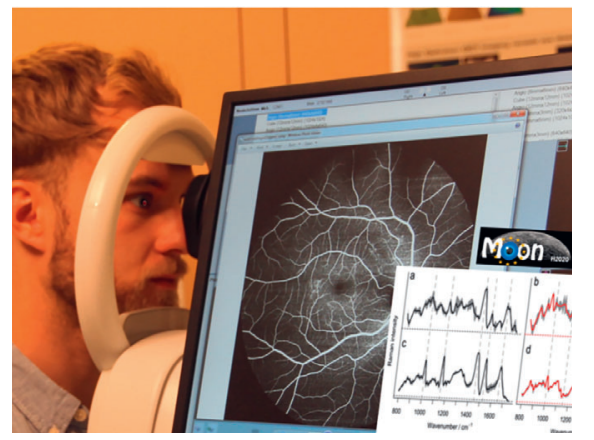

Das Forscherteam baut ein Gerät, an dem Patientinnen und Patienten ihr Auge berührungsfrei abrastern lassen können und wenige Minuten später eine Diagnose erhalten. (c) Ewald Unger/Medizinische Universität Wien

Immer mehr Menschen über 50 leiden an altersbedingten Sehstörungen. In vier von fünf Fällen wären diese nach Aussage der Weltgesundheitsorganisation vermeid- bar, wenn sie frühzeitig diagnostiziert würden. Ein europäisches Wissenschaftlerteam unter Beteiligung des Jenaer Leibniz-Instituts für Photonische Technologien (Leibniz-IPHT) hat nun eine neue Methode erforscht, mit der Mediziner solche Erkrankungen im Auge künftig besser erkennen können. Das optische Verfahren liefert mithilfe von Laserlicht in Sekundenschnelle detaillierte Informationen über den Zustand des Netzhautgewebes. Mit diesem Augenscan könnten Ärzte aggressive Formen von altersbedingter Makuladegeneration künftig eher entdecken und sogar neurodegenerativen Erkrankungen wie Alzheimer auf die Spur kommen.

Mithilfe markierungsfreier, molekular empfindlicher Raman-Spektroskopie gelingt es, einen molekularen Fingerabdruck der
Netzhaut zu gewinnen. So werden Veränderungen der Netzhaut sichtbar und Erkrankungen können in frühem Stadium erkannt werden.

Die Partner der Medizinischen Universität Wien bauen derzeit ein Gerät, das die Raman-Spektroskopie mit der OCT kombiniert. «So erhalten wir hochaufgelöste Bilder aus allen Schichten der Netzhaut mitsamt den Informationen über ihre molekulare Zusammensetzung», erläutert Prof. Jürgen Popp, wissenschaftlicher Direktor des Leibniz-IPHT. Dies könne die Genauigkeit der Diagnosen entscheidend verbessern.

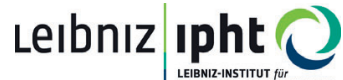

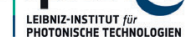 www.leibniz-ipht.de}

\section{Universität Greifswald \\ Erste Studie zur Behandlung der Frühgeborenenretinopathie mit VEGF-Inhibitoren veröffentlicht}

Die Frühgeborenenretinopathie stellt nach wie vor eine der häufigsten Erblindungsursachen bei Kindern weltweit dar. Die bisherige Standardtherapie der retinalen Laserkoagulation kann Nachteile wie beispielsweise Narbenbildung, Gesichtsfeldeinschränkungen und hohe Kurzsichtigkeit bei den behandelten Kindern mit sich bringen. In 26 Ländern wurde daher im Rahmen der RAINBOW-Studie untersucht, ob die Injektion des Medikaments Ranibizumab in das Auge erkrankter Kinder bessere Therapieergebnisse gegenüber der herkömmlichen Lasertherapie bietet. Die Ergebnisse dieser Studie wurden kürzlich online in der Fachzeitschrift THE LANCET publiziert.
Die Ergebnisse der Studie zeigen, dass die Therapie der Frühgeborenenretinopathie mit Ranibizumab sicher und effektiv ist. Auch wenn der statistisch signifikante Nachweis einer Überlegenheit von Ranibizumab gegenüber der Lasertherapie mit einem $p$-Wert von 0,051 ganz knapp verfehlt wurde, war der Anteil der Kinder mit erfolgreichem Behandlungsergebnis in der mit Ranibizumab 0,2 mg behandelten Gruppe mit 80\% deutlich höher als in der Lasergruppe (66\%). THE LANCET wählte hierfür die Formulierung «ranibizumab 0.2 mg might be superior to laser therapy». Insbesondere die Rate der unerwünschten strukturellen Veränderungen, die im späteren Leben das Sehvermögen behandelter Kin- der einschränken können, traten häufiger in der Lasergruppe auf (odds ratio $(\mathrm{OR})=2,19$ gegenüber Ranibizumab 0,2 mg).

Die im Rahmen der Studie behandelten Kinder werden aktuell in einer Folgestudie nachkontrolliert bis zu einem Alter von 5 Jahren, um auch Langzeitsicherheit und funktionelle Ergebnisse untersuchen zu können.

\section{Literatur}

1 Stahl A. et al. Lancet 2019. DOI: 10.1016/S01406736(19)31344-3.

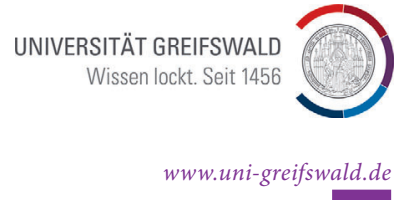




\section{Universität Hamburg \\ Sehvermögen im Kindesalter bestimmt Assoziationen zwischen Formen und Tönen}

Menschen fast aller Kulturen und Ethnien ordnen bestimmte Formen automatisch bestimmten Tönen zu. Wörter mit hoher Tonfrequenz (z.B. «Kiki») werden mit spitzen und eckigen Formen verknüpft, Wörter mit tiefer Tonfrequenz (z.B. «Bouba») vor allem mit glatten und runden Formen. Diese sogenannten multisensorischen Assoziationen wurden auch bei Ethnien beobachtet, die keine Schrift benutzen.

Das Team des Arbeitsbereichs Biologische Psychologie und Neuropsychologie der Universität Hamburg unter der Leitung von Prof. Dr. Brigitte Röder hat diese Zusammenhänge in einer neuen Studie untersucht. Dahinter steht die Frage nach sogenannten «sensiblen Phasen» der Entwicklung. In diesen Zeiträumen der Entwicklung ist das Gehirn besonders lernfähig. Nach ihrem Ablauf können manche Fähigkeiten nur noch sehr schwer oder gar nicht mehr erworben werden

Das Hamburger Team untersuchte Menschen, die aufgrund eines dichten Grauen Stars von Geburt an blind waren und erst spät operiert wurden. Außerdem nahmen Menschen teil, die vor dem 12. Lebensjahr Grauen Star bekommen hatten und für eine lange Zeit nicht oder nur sehr eingeschränkt sehen konnten, bevor sie ebenfalls operiert wurden. Ferner wurden blinde Menschen einbezogen, die entweder von Geburt an blind waren oder erst nach dem 12. Lebensjahr vollständig erblindet waren. Als Vergleichsgruppe dienten Menschen mit typischer Sehkraft.

Das Ergebnis: Probandinnen und Probanden, die von Geburt an blind waren, wiesen im Gegensatz zu den typisch sehenden Personen keine systematischen Form-TonAssoziationen auf. Auch Personen, die nach
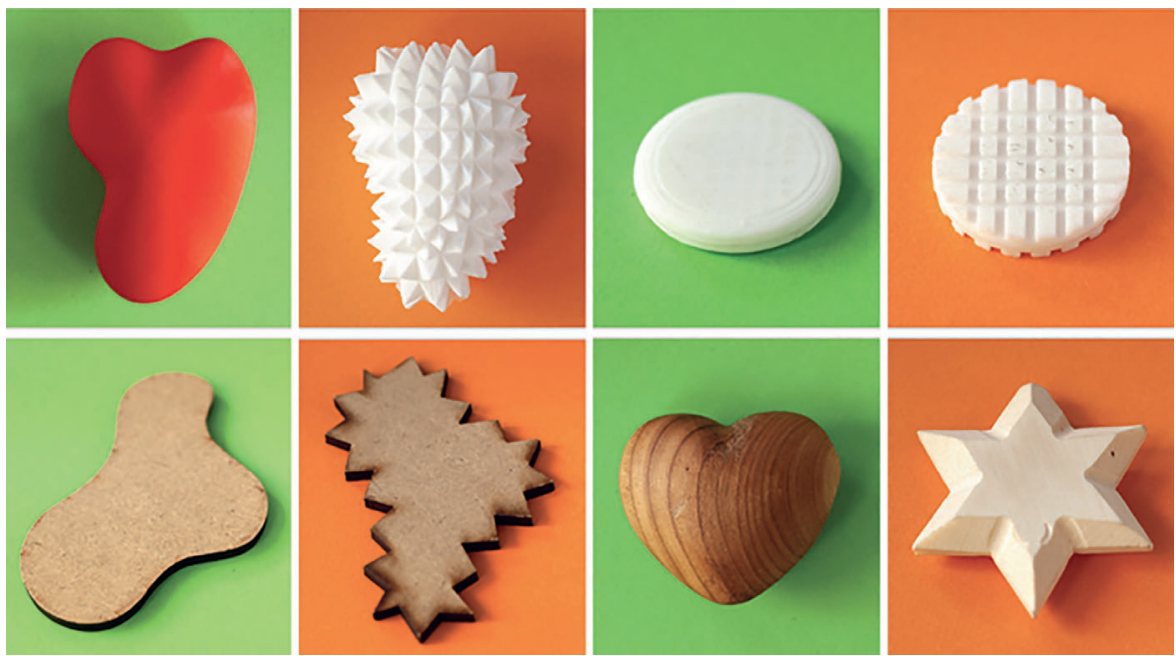

Menschen ordnen bestimmte Formen automatisch bestimmten Tönen zu. @ Nadine Esche

der Geburt oder in der frühen Kindheit eine Phase mit Blindheit oder starker Sehbehinderung durchlebt hatten, zeigten viele Jahre nach der Wiederherstellung des Augenlichts keine dieser Assoziationen.

Hinzu kam: Menschen, die für mindestens die ersten 12 Jahre normal gesehen hatten, bevor sie vollständig erblindeten, zeigten typische Assoziationen für ertastete Formen und Laute. Dies zeigt, dass Fähigkeiten, die während dieser Zeiten hoher Lernfähigkeit erworben werden, auch dann nicht verloren gehen, wenn sich die sensorische Wahrnehmung später verändert.

«Die Studie leistet einen wichtigen Beitrag zum Verständnis vom Zusammenspiel der verschiedenen Sinne und der besonderen Bedeutung der frühkindlichen Erfahrungen für unsere Hirnentwicklung», so Suddha Sourav, Erstautor der Studie.
In Zukunft möchten die Wissenschaftlerinnen und Wissenschaftler unter anderem genauer untersuchen, welche Gehirnmechanismen das Lernen nach Ende einer sensiblen Phase erschweren oder sogar verhindern. Gleichzeitig möchten sie erforschen, welche Hirnfunktionen, die nicht visuell sind, angesichts einer Blindheitsphase eine effizientere Verarbeitung aufweisen.

\section{Literatur}

1 Sourav S et al. Psychol Sci 2019;30(10): 1473-1482.

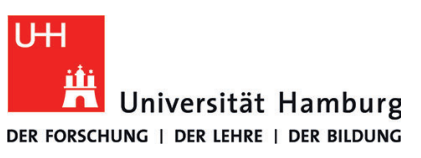

www.uni-hamburg.de 